\title{
Devil's Staircase Phase Diagram of the Fractional Quantum Hall Effect in the Thin-Torus Limit
}

\author{
Pietro Rotondo, ${ }^{1,2}$ Luca Guido Molinari, ${ }^{1,2}$ Piergiorgio Ratti, ${ }^{1}$ and Marco Gherardi ${ }^{1,3}$ \\ ${ }^{1}$ Dipartimento di Fisica, Università degli Studi di Milano, via Celoria 16, 20133 Milano, Italy \\ ${ }^{2}$ INFN Milano, via Celoria 16, 20133 Milano, Italy \\ ${ }^{3}$ Sorbonne Universités, UPMC Université Paris 06, UMR 7238, Computational and Quantitative Biology, \\ 15 rue de l'École de Médecine, 75006 Paris, France
}

(Received 2 August 2015; published 21 June 2016)

\begin{abstract}
After more than three decades, the fractional quantum Hall effect still poses challenges to contemporary physics. Recent experiments point toward a fractal scenario for the Hall resistivity as a function of the magnetic field. Here, we consider the so-called thin-torus limit of the Hamiltonian describing interacting electrons in a strong magnetic field, restricted to the lowest Landau level, and we show that it can be mapped onto a one-dimensional lattice gas with repulsive interactions, with the magnetic field playing the role of the chemical potential. The statistical mechanics of such models leads us to interpret the sequence of Hall plateaux as a fractal phase diagram whose landscape shows a qualitative agreement with experiments.
\end{abstract}

DOI: 10.1103/PhysRevLett.116.256803

The fractional quantum Hall effect (FQHE) [1] is among the most fascinating quantum phenomena involving strongly correlated electrons. It has attracted and fueled research in many directions since its discovery [2]. Lately, much interest has been directed toward investigating quantum Hall states as experimentally accessible prototypes of topological states of matter which have promising applications to quantum computation [3-5]. Currently, experiments in ultrahigh mobility 2D electron systems are revealing a fractal scenario for the Hall resistivity as a function of the magnetic field: indeed, more than 50 filling fractions are observed only in the lowest Landau level (LL) [6].

The physics of the FQHE is well understood phenomenologically thanks to the pioneering work by Laughlin and his celebrated ansatz for $1 / \mathrm{m}$ filling fractions [7]. The approach was generalized to more complicated fractions through the introduction of composite fermions [8,9] and a hierarchy of quasiparticles with fractional statistics [10-13], or by conformal invariance arguments [14-17]. A huge amount of results have been obtained over the years, confirming the validity of the approach based on model wave functions [2,18-20].

There is an ongoing effort toward the formulation of a systematic microscopic theory of the fractional quantum Hall effect. An intrinsic difficulty is the absence of an evident perturbative parameter, a common hindrance in strongly correlated systems [9]. In 1983 Tao and Thouless (TT) observed [21] that electrons in a strong magnetic field could form a one-dimensional Wigner crystal [22] in the lattice of degenerate states in the lowest LL, and they suggested that this mechanism may explain the fractional quantization of the Hall resistivity. However, the resulting many-body ground state displays long-range spatial correlations, in conflict with Laughlin's results. This route to a microscopic theory of the FQHE was abandoned (by Thouless himself [23]), as the Laughlin ansatz offers several advantages, e.g., its high overlap with the exact low-density ground state, and the fact that it constrains very naturally the filling fractions to have odd denominators. The TT framework was recently reconsidered by Bergholtz and his co-workers [24-26]. They found that TT states become the exact wave functions of the problem in the quasi-one-dimensional (thin-torus) limit.

Here, we study the thin-torus limit of the quantum Hall Hamiltonian in the lowest LL, and show that it realizes a repulsive gas on the lattice of degenerate Landau states, with the magnetic field acting as the inverse chemical potential. The zero-temperature statistical mechanics of this class of models was studied extensively [27-30]. It is characterized by an infinite series of second-order phase transitions, occurring at critical (nonuniversal) values of the chemical potential $\mu$. The density of particles $\rho(\mu)$ is the order parameter, and it takes a different rational value in each phase, thus producing a devil's staircase (a selfsimilar function with plateaux at rational values also known as the Cantor function) when plotted against $\mu$ [28]. There is a renewed interest in these models for potential applications to quantum simulators with ultracold Rydberg gases [31-34].

Our mapping allows us to (i) interpret the dependence of the transverse conductivity on the magnetic field as a fractal sequence of phase transitions, peculiar to $1 \mathrm{D}$ repulsive lattice gases, (ii) establish the incompressibility of the ground-state hierarchy in the thin-torus limit, and (iii) provide a theoretical prediction for the relative widths of different Hall plateaux. 
We consider the standard two-dimensional gas of $N_{e}$ interacting electrons in a uniform positive background, providing charge neutrality. We make the assumption that, in strong magnetic fields, the mixing between different Landau levels is suppressed; i.e., we work in the regime $e^{2} / \ell \ll \omega_{c}$, where $\ell=1 /(e B)^{1 / 2}$ is the magnetic length, $\omega_{c}=e B / m$ is the cyclotron frequency $(\hbar=c=1)$, and the spin degrees of freedom are frozen at the lowest spin level. We take the system to have area $L_{x} L_{y}$ and to be periodic in the $y$ direction, so that the single-particle wave functions may be written in the form

$$
\phi_{s}(x, y)=\left(\pi^{1 / 2} \ell L_{y}\right)^{-1 / 2} e^{-\left(2 \pi i s y / L_{y}\right)-(1 / 2)\left[(x / \ell)-\left(2 \pi s \ell / L_{y}\right)\right]^{2}},
$$

with $s=1,2, \ldots, N_{s}=L_{x} L_{y} / 2 \pi \ell^{2}$. The filling fraction $\nu=N_{e} / N_{s}$ is less than one.

In second quantization, the Coulomb interaction between the electrons in the lowest LL is

$$
H_{c}=\sum_{s_{1}, s_{2}, s_{3}=1}^{N_{s}} V_{s_{1}-s_{3}, s_{2}-s_{3}} a_{s_{1}}^{\dagger} a_{s_{2}}^{\dagger} a_{s_{1}+s_{2}-s_{3}} a_{s_{3}},
$$

where $a_{s}^{\dagger}, a_{s}$ are fermionic creation and annihilation operators, and momentum conservation in the periodic direction is manifest. The Coulomb matrix element can be parametrized in a useful form by considering periodic boundary conditions in both directions (torus geometry) $[21,35,36]$. See also the Supplemental Material [37].

$$
V_{s_{1}-s_{3}, s_{2}-s_{3}}=\frac{e^{2}}{L_{y}} \int_{-\infty}^{\infty} d q \frac{\exp \left[-\frac{\ell^{2}}{2}\left(q^{2}+\frac{4 \pi^{2}\left(s_{1}-s_{3}\right)^{2}}{L_{y}^{2}}\right)+\frac{2 \pi \mathrm{i} q \ell^{2}\left(s_{2}-s_{3}\right)}{L_{y}}\right]}{\sqrt{q^{2}+\frac{4 \pi^{2}\left(s_{1}-s_{3}\right)^{2}}{L_{y}^{2}}}} .
$$

The starting point of our analysis is the observation that, in the thin-torus limit $\ell / L_{x} \gg 1$, this matrix element depends on a single variable. The calculation (detailed in the SM [37]) shows that when it is nonzero, $V_{s_{1}-s_{3}, s_{2}-s_{3}}$ reduces to $W_{s_{1}-s_{3}} e^{2} / \ell$ (with $W_{s_{1}-s_{3}}$ being positive). By plugging this result into the Coulomb Hamiltonian, we obtain

$$
H_{c}=\frac{e^{2}}{\ell} \sum_{s_{1}, s_{2}, s} W_{s} a_{s_{1}+s}^{\dagger} a_{s_{2}-s}^{\dagger} a_{s_{2}} a_{s_{1}} .
$$

In the grand-canonical ensemble, the kinetic and chemical potential terms add up to an effective chemical potential. The total Hamiltonian is

$$
H_{L L L}=-\mu(B) \sum_{s=1}^{N_{s}} n_{s}+\frac{e^{2}}{\ell} \sum_{s_{1}, s_{2}, s} W_{s} a_{s_{1}+s}^{\dagger} a_{s_{2}-s}^{\dagger} a_{s_{2}} a_{s_{1}}
$$

where the definition $\mu(B)=\left(\tilde{\mu}-\omega_{c}\right)$ highlights the dependence of the effective chemical potential on the magnetic field.

Electrons in the lowest LL form a one-dimensional lattice (which we call target space). Importantly, they interact through a translationally invariant interaction (in the target space). The Hamiltonian is diagonalized in the Fourier basis, where the creation operator for the mode $k$ is $c_{k}^{\dagger}=1 / \sqrt{N_{s}} \sum_{s=1}^{N_{s}} e^{2 \pi \mathrm{i} k s / N_{s}} a_{s}^{\dagger}$. We obtain the following diagonal Hamiltonian with periodic boundary conditions:

$$
H_{L L L}=-\mu(B) \sum_{k=1}^{N_{s}} n_{k}+\frac{e^{2}}{\ell} \sum_{k_{1} \neq k_{2}} \tilde{W}\left(\left|k_{1}-k_{2}\right|\right) n_{k_{1}} n_{k_{2}}
$$

with $n_{k}=c_{k}^{\dagger} c_{k}$ and $\tilde{W}(k)=\sum_{s=1}^{N_{s}} e^{2 \pi \mathrm{i} k s / N_{s}} W(s)$ a repulsive potential. The explicit form of $\tilde{W}(k)$ is given in the SM [37]; it decays as $L_{x} /(\ell k)$.

The quantum Hall Hamiltonian on a torus $L_{x} \times L_{y}$ satisfies a notable duality relation, under the action of the unitary transformation $U$ to the Fourier modes. Denoting by $H_{c}(r)$ the Hamiltonian on a torus of aspect ratio $r=L_{x} / L_{y}$, one has (see the SM for the derivation [37])

$$
U^{\dagger} H_{c}(r) U=H_{c}(1 / r) .
$$

In this respect, our thin-torus limit is equivalent to the one usually considered in the literature [24].

The form (6) of the Hamiltonian realizes a mapping (in the thin-torus limit $L_{x} / \ell \ll 1$ ) of the FQHE on a onedimensional lattice gas with repulsive interactions whose degrees of freedom are the Fourier modes of the target space. As noted above, in these models the density, as a function of the chemical potential, exhibits a devil's staircase structure. Inspection of the Hamiltonian (6) shows that the role of the density is played by the filling fraction $\nu$, whereas the chemical potential can be tuned by the magnetic field $B$.

Schematically, the investigation of this class of models follows two steps. (i) The ground state of the system is sought at a fixed $\nu=p / q$ (with $p$ and $q$ being coprime); this problem was solved by Hubbard [38]; (ii) The stability region $\Delta \mu$ (under a single particle-hole exchange) of each ground state is determined; this was done by Bak and Bruinsma [28], and by Burkov and Sinai [30]. Both steps are subject to the technical condition that the potential be 
convex, which is fulfilled by the thin-torus potential $\tilde{W}(k)$. We reproduce this two-step construction in the following.

Intuitively, the ground state of a repulsive lattice gas at filling fraction $\nu=p / q$ is a configuration where particles are placed as far as possible from each other. The underlying lattice structure introduces the possibility of frustration, exhibited by deviations from the continuum equilibrium positions. The pattern of occupation numbers can be obtained through the continued-fraction expansion of $\nu=p / q$ :

$$
\frac{p}{q}=\frac{1}{n_{0}+\frac{1}{n_{1}+\frac{1}{\ddots \cdot+\frac{1}{n_{\lambda}}}}}
$$

Each level in the expansion realizes a better approximation of $\nu$; for a rational $\nu$, the number of levels $\lambda+1$ is finite. At $\lambda=0$ (i.e., $p=1$ ), the ground state is a periodic crystal with an interparticle distance $n_{0}=q$, corresponding to Laughlin-type states. At $\lambda=1$ the interparticle distances cannot be all equal, and a "defect" appears: the periodic ground state is formed by $\left(n_{1}-1\right)$ Laughlin-type blocks of density $1 / n_{0}$ and one block with density $1 /\left(n_{0}+1\right)$; these correspond to Jain-type states [a concise representation is $\left(n_{0}\right)^{n_{1}-1}\left(n_{0}+1\right)$ ]. This construction can be generalized iteratively to the level $\lambda$ (see Fig. 1 for three examples, as well as the SM [37]): the general rule uses the ground states at one level as building blocks to construct the ground states at the next level. The position of the $j$ th particle in the $\nu=p / q$ ground state can be expressed compactly as $\lfloor q / p j\rfloor$, where $\lfloor\cdot\rfloor$ denotes the integer part. (We notice en passant the connection with the sequences of characters known as Sturmian words).

Because of the periodic boundary conditions, the ground state at filling factor $\nu=p / q$ has a $q$-fold degeneracy, corresponding to the possible translations in the target space. This plays an important role when quantum effects are taken into account (see below). Summing up the

\begin{tabular}{|ccc|}
\hline$v$ & $C_{v}$ & Configuration \\
\hline$\frac{1}{3}$ & $(3)$ & $0000000000000 \cdots$ \\
$\frac{3}{7}$ & $(322)$ & $0000000000000 \cdots$ \\
$\frac{5}{13}$ & $(32)^{2}(3)$ & $0000000000000 \cdots$ \\
\hline
\end{tabular}

FIG. 1. Hubbard ground states for different filling fractions $\nu$ and their explicit periodic structure. The first two from the top belong, respectively, to the Laughlin $(\nu=1 / 3)$ and Jain $(\nu=3 / 7)$ series. Each periodic configuration may be expressed in a compact way through the sequence $C_{\nu}$ of its interparticle distances (the general algorithm to construct Hubbard ground states is extensively reviewed in the SM [37]). foregoing observations, a compact form of our ground states is the following:

$$
|\nu=p / q\rangle_{r}=\prod_{j=1}^{\left\lfloor p N_{s} / q\right\rfloor} c_{\lfloor q j / p\rfloor+r}^{\dagger}|0\rangle \quad r=0, \ldots, q-1 .
$$

We remark that the continued-fraction expansion that we employ to construct the ground states naturally provides a definition of "complexity" of a given filling fraction, via its level $\lambda$. This construction has a natural interpretation in terms of quasiparticles [39].

Once the ground states at general $\nu$ have been determined, their stability under a single particle-hole exchange can be established. The stability interval in the effective chemical potential is given by $[28,30]$

$$
\Delta \mu(p / q)=2 q \sum_{k=1}^{\infty} k(\tilde{W}(q k+1)+\tilde{W}(q k-1)-2 \tilde{W}(q k)) .
$$

As $\Delta \mu(\nu)>0$ for all rational filling fractions, this construction yields a phase diagram where each rational $\nu$ appears as the stable density for a finite interval of $\mu$ (hence of $B$ ), thus realizing a devil's staircase. As a consequence of our mapping, the stability equation (10) constitutes a proof of the incompressibility of the hierarchical ground states obtained in the thin-torus limit. It is worth remarking that the precise form of the potential does not affect qualitatively this result, as far as the convexity condition is fulfilled.

Even-denominator plateaux are not seen in the experiments in the lowest LL. This is not in contrast to formula (10), which holds for the ground states of the classical lattice gas. Indeed, the additional symmetry of the magnetic translation group realizes a selection rule preventing the appearance of even-denominator Hubbard states (an argument proposed by Seidel [40] is reviewed in the SM [37]). In the numerical computations below, we will assume that, when an even-denominator pattern is forbidden, the admissible ground state observed is gapless.

Our results support a new interpretation of the FQHE landscape (at least in the thin-torus limit) as the zerotemperature phase diagram of a fermionic one-dimensional lattice gas model with repulsive interactions. The results reported above allow us to plot a snapshot of the relation between the magnetic field and the inverse filling fraction. The potential $\tilde{W}$ has a nontrivial dependence on the magnetic length $\ell$. As noted above, it scales as $1 / \ell$. Therefore, by rescaling the entire Hamiltonian, $H \rightarrow H \ell^{2}$, the whole dependence on the magnetic field $B$ is absorbed in the chemical potential $\mu(B) \ell^{2}$, which now depends on $B^{-1}$ :

$$
\mu=\tilde{\mu} /(e B)-1 / m .
$$

Operatively, we set a cutoff $q_{\max }$ on the possible denominators, we list (in increasing order) all filling fractions $p / q$ 


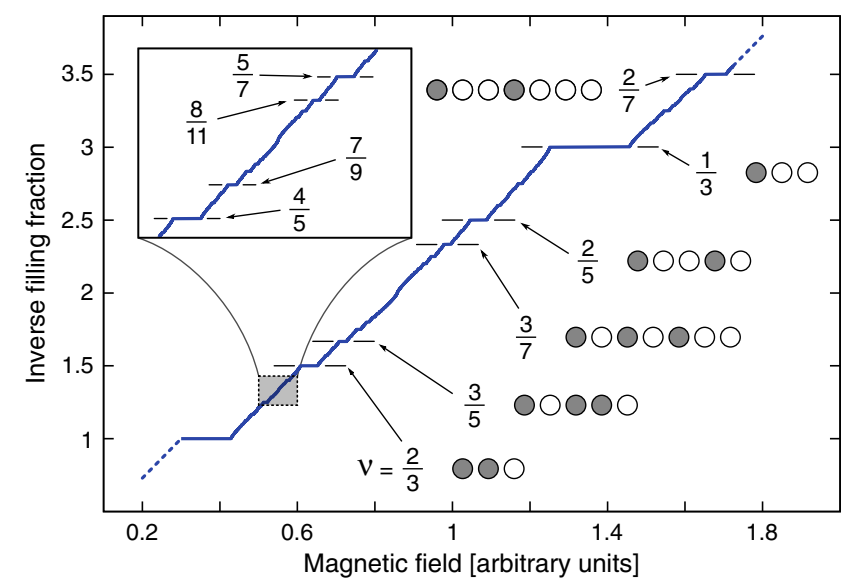

FIG. 2. Inverse filling fraction $1 / \nu$ plotted against the magnetic field $B$ (in arbitrary units). The most visible plateaux are highlighted with their corresponding occupational periodic pattern in the reciprocal target space. This snapshot shows a qualitative agreement with the experimental measures of Hall resistivity, both for the relative widths of the plateaux and for the quasilinear trend of the landscape as a function of $B$. (Inset) A portion of the staircase is magnified and some experimentally observed plateaux [6] are marked.

such that $q$ is odd, $q \leq q_{\max }$, and $p=1, \ldots, q$, and we compute $\Delta \mu$ for each one of them. (Only odd denominators are considered because of the assumption above.) Doing this by increasing order allows us to obtain iteratively the two stability boundaries, $\mu_{-}$and $\mu_{+}$, of each plateau; the corresponding values of the magnetic field, $B_{-}$and $B_{+}$, are calculated from the relation (11). The resulting landscape, presented in Fig. 2, is qualitatively in accord with the well-known behavior obtained in experiments.

The values of the numerators and the denominators in the filling fractions have two different effects on the plateau widths. Equation (10) shows that the width of a plateau as a function of the chemical potential $\mu$ only depends on the denominator. Specifically, the plateaux get narrower as the denominator $q$ is increased [as can be checked by plugging $\tilde{W} \sim 1 / k$ into Eq. (10)], and filling fractions with the same denominator have the same $\Delta \mu$. Therefore, the inverse filling fraction as a function of $\mu$ is a staircase, symmetric in the particle-hole exchange; i.e., filling fractions $\nu$ and $1-\nu$ have the same width, $\Delta \mu$. However, the chemical potential is a function of the inverse magnetic field [Eq. (11)]. This nonlinear relation between $\mu$ and $B$ breaks the symmetry, thus enhancing the stability of plateaux at larger magnetic fields. As a consequence, filling fractions with the same denominator have larger stability intervals (in $B$ ) for smaller numerators $p$. The most evident example of this general mechanism can be recognized in the fact that the plateau at $\nu=1 / 3$ is larger than that at $\nu=2 / 3$, as is experimentally observed. Summing up, the relative widths of the plateaux are determined by two different contributions: the numerator-independent width given by Eq. (10) and the deformation of the $B$ axis due to the relation $\mu(B)$.

In statistical mechanics, systems with slowly decaying potentials are pathological: their free energy is not extensive as a function of the particle number. In our framework, this has the effect of pushing the staircase toward infinity as the cutoff $q_{\max }$ is increased. This issue may be overcome by regularizing the Coulomb potential. Our thin-torus analysis is largely independent of the precise form of the potential, while the relative widths of the plateaux depend on it. The simple mechanism for the breaking of the electron-hole symmetry, here presented for the Coulomb potential, remains valid for potentials with the periodicity of the torus and $\tilde{v}(q)=C q^{-1-\alpha}$ (this is shown in the SM [37]). For general potentials, the interaction Hamiltonian may have a dependence on the magnetic field $B$ that does not factorize, making a simple redefinition of $\mu$ not viable. These cases require a more general analysis that we do not pursue here.

The main result of this work is the mapping between the Hall Hamiltonian in the thin-torus limit and a long-range repulsive lattice gas model in one dimension. This result allows us to interpret the FQH ground states as Hubbard states, and to prove their incompressibility, as a direct consequence of Eq. (10). The lattice gas also brings us to a scenario where the Hall resistivity as a function of the magnetic field is a devil's staircase. By assuming that evendenominator ground states are gapless, qualitative accordance with the experimental landscape is obtained. This suggests that it may be fruitful to investigate the nature of the correlated ground states at more exotic fillings in the lowest LL. This is possible, in principle, by generalizing the composite-fermion picture (recently used to propose new incompressible ground states at $\nu=4 / 11$ and $\nu=5 / 13$ [41]), or by exploiting the recent results with Jack polynomials $[16,42,43]$.

We are grateful to Bruno Bassetti, Sergio Caracciolo, Mario Raciti, Marco Cosentino Lagomarsino, Andrea Sportiello, and Alessio Celi for the useful discussions and advice.

[1] D. C. Tsui, H. L. Stormer, and A. C. Gossard, Phys. Rev. Lett. 48, 1559 (1982).

[2] H. L. Stormer, Rev. Mod. Phys. 71, 875 (1999).

[3] C. Nayak, S. H. Simon, A. Stern, M. Freedman, and S. D. Sarma, Rev. Mod. Phys. 80, 1083 (2008).

[4] A. Y. Kitaev, Ann. Phys. (Amsterdam) 303, 2 (2003).

[5] A. Stern, Ann. Phys. (Amsterdam) 323, 204 (2008).

[6] W. Pan, J. S. Xia, H. L. Stormer, D. C. Tsui, C. Vicente, E. D. Adams, N. S. Sullivan, L. N. Pfeiffer, K. W. Baldwin, and K. W. West, Phys. Rev. B 77, 075307 (2008).

[7] R. B. Laughlin, Phys. Rev. Lett. 50, 1395 (1983).

[8] J. K. Jain, Phys. Rev. Lett. 63, 199 (1989). 
[9] J. K. Jain, Composite Fermions (Cambridge University Press, Cambridge, England, 2007).

[10] F. D. M. Haldane, Phys. Rev. Lett. 51, 605 (1983).

[11] B. I. Halperin, Phys. Rev. Lett. 52, 1583 (1984).

[12] F. Wilczek, Phys. Rev. Lett. 49, 957 (1982).

[13] F. D. M. Haldane, Phys. Rev. Lett. 67, 937 (1991).

[14] G. Moore and N. Read, Nucl. Phys. B360, 362 (1991).

[15] N. Read and E. Rezayi, Phys. Rev. B 59, 8084 (1999).

[16] B. A. Bernevig and F. D. M. Haldane, Phys. Rev. Lett. 100, 246802 (2008).

[17] E. Bettelheim, I. A. Gruzberg, and A. W. W. Ludwig, Phys. Rev. B 86, 165324 (2012).

[18] A. C. Balram, A. Wójs, and J. K. Jain, Phys. Rev. B 88, 205312 (2013).

[19] W. Pan, H. L. Stormer, D. C. Tsui, L. N. Pfeiffer, K. W. Baldwin, and K. W. West, Phys. Rev. Lett. 90, 016801 (2003).

[20] J. K. Jain, Indian J. Phys. 88, 915 (2014).

[21] R. Tao and D. J. Thouless, Phys. Rev. B 28, 1142 (1983).

[22] E. Wigner, Phys. Rev. 46, 1002 (1934).

[23] D. J. Thouless, Phys. Rev. B 31, 8305 (1985).

[24] E. J. Bergholtz, T. H. Hansson, M. Hermanns, and A. Karlhede, Phys. Rev. Lett. 99, 256803 (2007).

[25] E. J. Bergholtz and A. Karlhede, Phys. Rev. B 77, 155308 (2008).

[26] E. J. Bergholtz and A. Karlhede, Phys. Rev. Lett. 94, 026802 (2005).

[27] P. Bak, Rep. Prog. Phys. 45, 587 (1982).
[28] P. Bak and R. Bruinsma, Phys. Rev. Lett. 49, 249 (1982).

[29] S. Aubry, J. Phys. C 16, 2497 (1983).

[30] S. Burkov and Y. G. Sinai, Russ. Math. Surv. 38, 235 (1983).

[31] P. Schauß, J. Zeiher, T. Fukuhara, S. Hild, M. Cheneau, T. Macrì, T. Pohl, I. Bloch, and C. Gross, Science 347, 1455 (2015).

[32] Z. Xu, L. Li, and S. Chen, Phys. Rev. Lett. 110, 215301 (2013).

[33] E. Levi, J. Minář, and I. Lesanovsky, J. Stat. Mech. (2016) 033111.

[34] E. Levi, J. Mináŕ, J. P. Garrahan, and I. Lesanovsky, arXiv:1503.03259.

[35] D. Yoshioka, B. I. Halperin, and P. A. Lee, Phys. Rev. Lett. 50, 1219 (1983).

[36] D. Yoshioka, Phys. Rev. B 29, 6833 (1984).

[37] See Supplemental Material at http://link.aps.org/ supplemental/10.1103/PhysRevLett.116.256803 for the details on the derivation of the equations in the main text.

[38] J. Hubbard, Phys. Rev. B 17, 494 (1978).

[39] V. Pokrovsky and G. Uimin, J. Phys. C 11, 3535 (1978).

[40] A. Seidel, Phys. Rev. Lett. 105, 026802 (2010).

[41] S. Mukherjee, S. S. Mandal, Y.-H. Wu, A. Wójs, and J. K. Jain, Phys. Rev. Lett. 112, 016801 (2014).

[42] B. A. Bernevig and N. Regnault, Phys. Rev. Lett. 103, 206801 (2009).

[43] R. Thomale, B. Estienne, N. Regnault, and B. A. Bernevig, Phys. Rev. B 84, 045127 (2011). 